\title{
Modelling of the behaviour of metal foams under shock compression
}

\author{
Nicolas Jacques ${ }^{1, *}$ and Romain Barthélémy ${ }^{2}$ \\ ${ }^{1}$ ENSTA Bretagne, FRE CNRS 3744, IRDL, 2 rue François Verny, F-29806 Brest cedex 9, France \\ ${ }^{2}$ MBDA France, 1 avenue Réaumur, F-93350 Le Plessis-Robinson, France
}

\begin{abstract}
A theoretical modelling is proposed to describe the shock response of foam materials. This model is based on micromechanical and energetic arguments, and takes into account the contribution of microscale inertia. Within this framework, an analytical expression of the Hugoniot stress-strain curve is proposed for elastic-plastic cellular materials. The predictions derived from the proposed model are in excellent agreement with experimental data for open-cell aluminium foams. The case of viscoplastic foams is also considered.
\end{abstract}

\section{Introduction}

Foams and cellular materials are highly porous media with a relative density generally lower than 0.3 . They have interesting mechanical properties for shock and impact applications due to their ability to undergo large compressive strains. The dynamic compressive behaviour of cellular solids has been the subject of numerous investigations, see e.g. the recent review of Sun et al. [1]. It is well known that foams are susceptible to form shock waves at moderate impact speed (generally lower than $100 \mathrm{~m} / \mathrm{s}$ ). The formation of a shock wave in a foam specimen yields significant (macroscopic) inertia effects and an apparent enhancement of the crushing response [2]. Moreover, several recent studies, based either on experiments [3] or cell-based numerical simulations [4-6], have shown that the stress-strain relation of foams under shock loading is significantly different from their quasi-static compressive response. Different mechanisms can affect the shock response of cellular solids. Firstly, the macroscopic rate-sensitivity of foams can be inherited from rate-dependent properties of the base material (material rate-sensitivity). Secondly, the rapid crushing of the cells of the foam microstructure can generate local inertia effects (micro-inertia effects), that can affect the macroscopic behaviour [7-8].

The aim of the present work is to develop a continuum model able to describe the behaviour of cellular materials under shock loading. The proposed model is based on a micromechanical analysis and takes macroscopic and microscopic inertia into account. In the case of elastic-plastic cellular solids (made of a rateindependent base material), an analytical expression of the Hugoniot stress-strain curve was obtained. A method to determine the Hugoniot and the structure of shock waves in viscoplastic foams is also proposed.

\section{Continuum modelling of the shock response of cellular materials}

\subsection{Theoretical background: micromechanics of cellular solids under dynamic loading}

Micro-inertia effects in foam materials are described using a Lagrangian dynamic homogenisation procedure. This procedure is briefly described in this section. More details can be found in [9-10].

We consider a Representative Volume Element (RVE) of a heterogeneous solid. Let $x_{i}$ and $x_{j}^{0}(i, j=$ $1,2,3)$ be the components in a Newtonian coordinate system of the current and initial positions of a given material point of the RVE. The microscopic deformation gradient $\partial x_{i} / \partial x_{j}^{0}$ is denoted by $f_{i j}$. Inertia effects are taken into account at the scale of the RVE. Therefore, the microscopic nominal stress tensor $p_{j i}$ satisfies the equation of balance of linear momentum:

$$
\frac{\partial p_{j i}}{\partial x_{j}^{0}}=\rho_{0} \gamma_{i}
$$

with $\gamma_{i}$ being the microscopic acceleration field.

The dynamic virtual work principle is used to link the microscopic and macroscopic scales. The macroscopic nominal stress $P_{j i}$ is related to the microscopic stress and acceleration fields by the following equation:

$$
\delta F_{i j} P_{j i}=\left\langle\delta f_{k m} p_{m k}\right\rangle+\left\langle\rho_{0} \delta u_{k} \gamma_{k}\right\rangle
$$

where $\delta F_{i j}$ denotes a virtual variation of the macroscopic deformation gradient and the brackets stand for the volume average operator. We consider a kinematically admissible displacement field having the following form:

$$
u_{k}=u_{k}\left(x_{m}^{0}, F_{i j}\right)
$$

Combining Eqs. (2) and (3), one can obtain the following expression of the macrostress: 


$$
P_{j i}=\left\langle L_{k m i j} p_{m k}\right\rangle+\left\langle\rho_{0} K_{k i j} \gamma_{k}\right\rangle=P_{j i}^{s t a}+P_{j i}^{d y n}
$$
with $\mathrm{L}_{k m i j}=\partial^{2} u_{k} / \partial x_{m}^{0} \partial F_{i j}$ and $\mathrm{K}_{k i j}=\partial u_{k} / \partial F_{i j}$. The macrostress involves two contributions. This first one, $P_{j i}^{s t a}$, is a static stress related to the microscopic stress field and therefore to the material response of the base material of the RVE. The second contribution, $P_{j i}^{d y n}$, is a dynamic stress due to microscale inertia effects.

An interesting property of the dynamic stress is its relation to (microscale) kinetic energy. The kinetic energy density (energy per unit initial volume) due to micro-inertia is given by

$$
K_{\mu}=\frac{1}{2}\left\langle\rho_{0} v_{k} v_{k}\right\rangle
$$

The microscopic velocity $v_{k}$ is related to the time derivative of the macroscopic deformation gradient by the following equation

$$
v_{k}=\mathrm{K}_{k i j} \dot{F}_{i j}
$$

Differentiating Eq. (5) with respect to time, one obtains

$$
\dot{K}_{\mu}=\left\langle\rho_{0} \gamma_{k} v_{k}\right\rangle=\left\langle\rho_{0} K_{k i j} \gamma_{k}\right\rangle \dot{F}_{i j}
$$

From Eqs. (4) and (7), it appears that the dynamic stress is directly related to the time evolution of the microkinetic energy density:

$$
\dot{K}_{\mu}=P_{j i}^{d y n} \dot{F}_{i j}
$$

\subsection{Analytical expression of the Hugoniot curve for elastic-plastic cellular materials}

In the present section, an analytical expression of the Hugoniot stress-strain curve is developed. We consider the propagation of a steady-state planar shock along the $X_{1}$-axis. In an elastic-plastic solid, a shock wave is preceded by an elastic precursor and the compressive stress ahead of the shock front is equal to $\Sigma_{0}$ the initial yield stress of the material [3]. Let us denote by $P_{H}$ and $\varepsilon_{H}$, respectively, the compressive stress and strain, respectively, behind the shock front. These quantities are linked by the Hugoniot curve that describes the shock response of the material (the Hugoniot defines all possible shocked states in the material).

The macroscopic balance of momentum and continuity equations for the cellular solid can be written as

$$
\begin{aligned}
& \frac{\partial P_{11}^{c}}{\partial X_{1}^{0}}=-\overline{\rho_{0}} \frac{\partial^{2} U_{1}}{\partial t^{2}} \\
& \varepsilon_{11}^{c}=-\frac{\partial U_{1}}{\partial X_{1}^{0}}
\end{aligned}
$$

where $P_{11}^{c}$ and $U_{1}$ are respectively the components of the compressive stress $\left(P_{11}^{c}=-P_{11}\right)$ and of the displacement in the shock propagation direction and $\overline{\rho_{0}}$ is the average initial mass density of the cellular solid. $\varepsilon_{11}^{c}$ is the nominal compressive strain. We consider that the shock wave travels at a constant celerity denoted by $C_{S}$. Therefore, spatial and time derivatives are related by the following equation:

$$
\frac{\partial \mathbf{m}}{\partial t}=C_{S} \frac{\partial \mathbf{m}}{\partial X_{1}^{0}}
$$

From Eqs. (9-11), the following relationship is obtained

$$
\frac{\partial P_{11}^{c}}{\partial X_{1}^{0}}=\overline{\rho_{0}} C_{S}{ }^{2} \frac{\partial \varepsilon_{11}^{c}}{\partial X_{1}^{0}}
$$

Integration of this equation yields

$$
P_{11}^{c}-\Sigma_{0}=\overline{\rho_{0}} C_{S}^{2}\left(\varepsilon_{11}^{c}-\varepsilon_{0}\right)
$$

where $\varepsilon_{0}$ is the compressive strain ahead of the shock front (corresponding to the initial yield strain of the material). From Eq. (13), it appears that the response of the material during the shock compaction corresponds to a linear path joining the state ahead of the shock front $\left(\Sigma_{0}, \varepsilon_{0}\right)$ to the state behind the shock front $\left(P_{H}, \varepsilon_{H}\right)$. This linear evolution of the stress is known as the Rayleigh line. The end point of the Rayleigh line is a point of the Hugoniot curve.

According to the framework presented in section 2.1, the compressive stress is the sum of a static and a dynamic component, $P_{11}^{c}=P_{11}^{c \text { sta }}+P_{11}^{c d y n}$. In the present section, we consider that the base material of the cellular solid has a rate-independent behaviour (this is for instance the case for some aluminium foams [11]). Thus, the static stress is assumed to be only dependent on strain (and not on strain rate), $P_{11}^{c \text { sta }}=P_{11}^{c \text { sta }}\left(\varepsilon_{11}^{c}\right)$. However, it should be noted that the macroscopic response of the cellular solid remains rate-dependent because of the dynamic stress [9].

As discussed in the previous section, there is a relation between dynamic stress and kinetic energy. In the context of planar shock propagation, Eq. (8) reduces to

$$
\dot{K}_{\mu}=P_{11}^{c d y n} \cdot \dot{\varepsilon}_{11}^{c}
$$

It is worth emphasizing that the kinetic energy density due to micro-inertia $K_{\mu}$ is only related to the deformation of the material and is independent to the macroscopic kinetic energy related to the macroscopic velocity field $V_{1}$. Indeed, the microscopic velocity $v_{k}$ corresponds to the velocity relative to the centre of mass of the RVE $[9,12]$. As a consequence, the micro-kinetic energy density $K_{\mu}$ vanishes at the end of the shock compression when the strain reaches its final value $\varepsilon_{H}$. From Eq. (14), this implies that the work of the dynamic stress during the shock compaction is equal to zero:

$$
\begin{gathered}
\int_{\varepsilon_{0}}^{\varepsilon_{H}} P_{11}^{c d y n} \cdot \mathrm{d} \varepsilon_{11}^{c}=0 \\
\text { As } P_{11}^{c}=P_{11}^{c s t a}+P_{11}^{c d y n} \text {, it follows that } \\
\int_{\varepsilon_{0}}^{\varepsilon_{H}} P_{11}^{c} \cdot \mathrm{d} \varepsilon_{11}^{c}=\int_{\varepsilon_{0}}^{\varepsilon_{H}} P_{11}^{c s t a t} \cdot \mathrm{d} \varepsilon_{11}^{c}
\end{gathered}
$$

Considering the Rayleigh line equation (13), we finally obtain the following expression of the Hugoniot stress

$$
P_{H}=\frac{2}{\left(\varepsilon_{H}-\varepsilon_{0}\right)} \int_{\varepsilon_{0}}^{\varepsilon_{H}} P_{11}^{c s t a} \cdot \mathrm{d} \varepsilon_{11}^{c}-\Sigma_{0}
$$

For an elastic-plastic solid (made of a rate-insensitive base material), the static stress $P_{11}^{c s t a}$ depends only on the compressive strain $\varepsilon_{11}^{c}$. Therefore, the application of Eq. (17) is straightforward and the Hugoniot curve $\left(P_{H}-\varepsilon_{H}\right.$ relation) can be determined from the static response of the cellular material. It is also interesting to notice that for the derivation of Eq. (17), we did not consider a specific expression of the dynamic stress. This means that the expression of the Hugoniot curve is not related to a specific RVE and micro-inertia model. To obtain Eq. (17), we have only used the property that the work of the dynamic stress during the shock compression is equal to zero, see Eq. (15). This is a general property of the constitutive framework presented in section 2.1, valid regardless of the RVE geometry. This property is due to the fact that the kinetic energy due to micro-inertia vanishes at the end of the shock 
compression (because the material no longer deforms). Consequently, Eq. (17) can be applied to any kind on cellular material.

\subsection{Computation of the Hugoniot of viscoplastic closed-cell foams}

In the present section, the case of viscoplastic foams, for which the static stress is rate-dependent, $P_{11}^{c \text { sta }}=$ $P_{11}^{c s t a}\left(\varepsilon_{11}^{c}, \dot{\varepsilon}_{11}^{c}\right)$, is considered. It should be noted that Eq. (17) remains valid for a viscoplastic material. However, in this case, the determination of the strain-rate history during the shock compression is needed to evaluate the Hugoniot stress. It is expected that both viscoplastic and micro-inertia effects influence the shock structure [1314]. The selection of a specific micro-inertia model is therefore necessary. The model proposed in [9] for closed-cell foams is considered. The dynamic stress in the shock propagation direction reads

$$
P_{11}^{c d y n}=\frac{1}{3} \bar{\rho}_{0} R_{c}{ }^{2} \ddot{\varepsilon}_{11}^{c}
$$

where $R_{c}$ is the radius of the cells of the foam microstructure. Hence, the total stress is given by

$$
P_{11}^{c}=P_{11}^{c s t a}\left(\varepsilon_{11}^{c}, \dot{\varepsilon}_{11}^{c}\right)+\frac{1}{3} \overline{\rho_{0}} R_{c}{ }^{2} \ddot{\varepsilon}_{11}^{c}
$$

Combining this expression with the Rayleigh line equation (13), the following differential equation is obtained

$$
\ddot{\varepsilon}_{11}^{c}=\frac{3}{R_{C}{ }^{2}}\left(C_{S}{ }^{2}\left(\varepsilon_{11}^{c}-\varepsilon_{0}\right)+\frac{\Sigma_{0}-P_{11}^{c s t a}\left(\varepsilon_{11}^{c}, \dot{\varepsilon}_{11}^{c}\right)}{\overline{\rho_{0}}}\right)
$$

This equation can be integrated numerically (using e.g. a second-order Runge-Kutta scheme). For a given shock celerity $C_{S}$, the integration is carried out until the strainrate tends to zero (indicating the end of the shock compaction). The final values of $P_{11}^{c}$ and $\varepsilon_{11}^{c}$ correspond to a point of the Hugoniot. The Hugoniot curve is constructed by considering several values of $C_{S}$. It has been checked that, for foams with no material ratesensitivity, the Hugoniot obtained by numerical integration of Eq. (20) is identical to the one derived from the analytical model presented in section 2.2 (Eq. (17)).

It should be noted that integration of Eq. (20) also provides the shock wave structure. Indeed, for each time, there is a correspondence with a Lagrangian position according to $\mathrm{d} X_{1}^{0}=C_{S} \cdot \mathrm{d} t$.

\section{Results}

\subsection{Comparison with experimental data for open-cell aluminium foams}

In the present section, the predictions of the proposed continuum modelling are compared to the experimental results of Barnes et al. [3]. These researchers performed impact tests to characterize the shock response of Duocel $^{\circledR}$ aluminium open-cell foam specimens. The shock stress was measured using a pressure bar while the strain behind the shock front was obtained from highspeed photographic sequences using digital image correlation. The experimental technique employed by
Barnes et al. [3] allowed a direct determination of the Hugoniot stress-strain curve of the foam.

From two quasi-static crushing curves available in [3] (labelled QS-2 and QS-3 in Fig. 1), the following relationship was identified to describe the static response of the foam:

$$
P_{11}^{c s t a}\left(\varepsilon_{11}^{c}\right)=\Sigma_{0}+\frac{A \cdot\left(\varepsilon_{11}^{c}-\varepsilon_{0}\right)}{\left(\varepsilon_{11}^{c}-\varepsilon_{0}-\varepsilon_{L}\right)^{2}}
$$

with $\varepsilon_{0}=0.0125, \quad \Sigma_{0}=2.3 \mathrm{MPa}, A=0.259 \mathrm{MPa}$ and $\varepsilon_{L}=0.879$. Duocel aluminium foams have generally no material strain-rate sensitivity [11]. Therefore, the analytical model described in section 2.2 (Eq. (17)) was used to compute the Hugoniot curve from the static response.

The calculated Hugoniot is compared to the measurements of Barnes et al. [3] in Fig. 1. A very good agreement between theory and experiments is observed. From Fig. 1, it is also interesting to notice the difference between the Hugoniot curve and the static response. For a given stress level, the Hugoniot strain is significantly larger the static crushing strain. This means that a shock loading leads to a stronger densification of the foam. A similar behaviour was observed in [9] and was found to be related to a release of micro-kinetic energy at the end of the foam deformation.

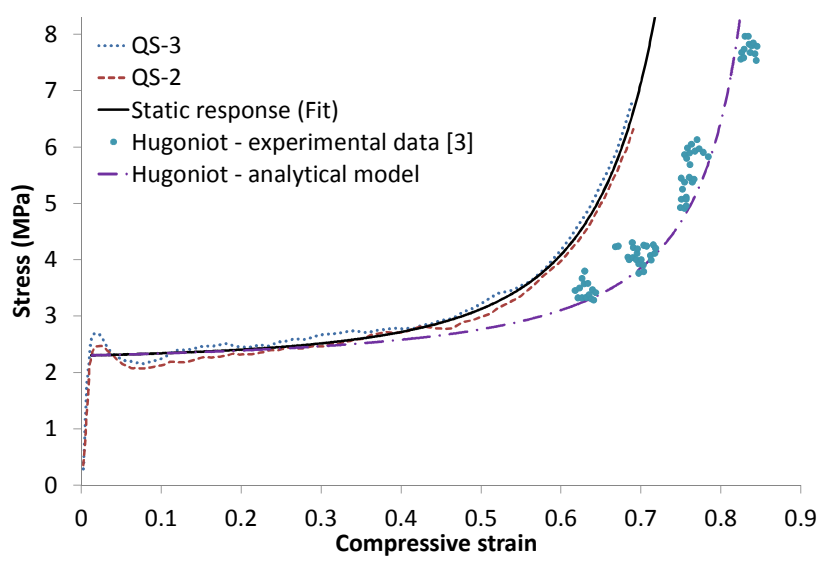

Fig. 1. Comparison between the Hugoniot obtained with the proposed analytical model (Eq. (17)) and the experimental data of Barnes et al. [3]. The static foam response is also showed.

\subsection{Hugoniot and shock behaviour of viscoplastic foams}

We now turn our attention to the shock behaviour of viscoplastic foams. We consider the following relationship to describe the static (aninertial) foam response:

$$
\begin{gathered}
P_{11}^{c s t a}\left(\varepsilon_{11}^{c}, \dot{\varepsilon}_{11}^{c}\right)=P_{11}^{c R I}\left(\varepsilon_{11}^{c}\right)+P_{11}^{c \text { vis }}\left(\dot{\varepsilon}_{11}^{c}\right) \\
P_{11}^{c R I}\left(\varepsilon_{11}^{c}\right)=\Sigma_{0}+\frac{A \cdot\left(\varepsilon_{11}^{c}-\varepsilon_{0}\right)}{\left(\varepsilon_{11}^{c}-\varepsilon_{0}-\varepsilon_{L}\right)^{2}} \\
P_{11}^{c v i s}\left(\dot{\varepsilon}_{11}^{c}\right)=\alpha \Sigma_{0}\left(\frac{\dot{\varepsilon}_{11}^{c}}{\dot{\varepsilon}_{0}}\right)^{m}
\end{gathered}
$$

With this model, the static stress is written as the sum of a rate-independent stress $P_{11}^{c R I}$ (corresponding to the foam response at vanishing strain-rate) and a viscous 
stress $P_{11}^{c \text { vis }}$. The following parameters have been adopted: $\varepsilon_{0}=0.008, \quad \Sigma_{0}=6.3 \mathrm{MPa}, A=0.2625 \mathrm{MPa}$, $\varepsilon_{L}=0.935, \dot{\varepsilon}_{0}=1000 \mathrm{~s}^{-1}, m=0.1, R_{c}=1.65 \mathrm{~mm}$ and $\frac{\varepsilon_{L}}{\rho_{0}}=277 \times 10^{-12} \mathrm{t} / \mathrm{mm}^{3}$. Several values of the parameter $\alpha$ have been considered to study the influence of material rate-sensitivity on the shock foam behaviour: $\alpha=0$ (no rate-sensitivity), $\alpha=0.1$ and $\alpha=0.5$. The computations have been carried out with the method presented in section 2.3.

Fig. 2 presents the Hugoniot curves calculated for the three values of the parameter $\alpha$. The foam response at low strain-rate (given by $P_{11}^{c R I}\left(\varepsilon_{11}^{c}\right)$ ) is also displayed for the sake of comparison. It can be observed that, under a certain shock stress magnitude, the Hugoniot is identical to the low strain-rate response of the foam. With $\alpha=$ 0.5 , this occurs for $P_{H}$ lower than $15 \mathrm{MPa}$. When the shock stress goes beyond this threshold, the Hugoniot gradually deviates from the low-strain rate curve. For very large shock stresses $\left(P_{H}>30 \mathrm{MPa}\right)$, the Hugoniot curves obtained for the three values of $\alpha$ are almost identical. This means that the influence of material ratesensitivity becomes negligible for strong shocks.

The behaviour of viscoplastic foams under shock compaction is dominated by viscous effects for low shock magnitudes, while micro-inertia effects become predominant at high amplitude. This point is illustrated in Fig. 3, which displays the constitutive response of a viscoplastic foam $(\alpha=0.5)$ for two shock magnitudes $\left(P_{H}=14 \mathrm{MPa}\right.$ and $\left.P_{H}=21.5 \mathrm{MPa}\right)$. The evolutions of the total stress, the rate-independent stress, the viscous stress and the dynamic stress are plotted. It is reminded that, during a shock wave compression, the total stress follows a linear evolution (the Rayleigh line) and that the difference between the total stress and the rateindependent stress is equal to the sum of the viscous and dynamic stress. From Fig. 3-a, it can be observed that, for the lowest shock amplitude $\left(P_{H}=14 \mathrm{MPa}\right)$, the dynamic stress remains small as compared to the viscous stress. Moreover, the dynamic stress vanishes in the late stage of the deformation. For this reason, the deformation ends when the Rayleigh line intersects the rate-independent compression curve. The same does not occur for $P_{H}=21.5 \mathrm{MPa}$ (Fig. 3-b). In this case, the maximum value of the dynamic stress is of the same order than the maximum viscous stress. In the late stage of deformation, the dynamic stress becomes negative. The sign of the dynamic stress is related to the evolution of the micro-kinetic energy density, see Eq. (14). The negative values of $P_{11}^{c d y n}$ observed in Fig. 3-b for $\varepsilon_{11}^{c}>0.77$ corresponds to a release of microscale kinetic energy. Owing to this release of micro-kinetic energy, the deformation continues after that the Rayleigh line crosses the rate-independent stress-strain curve.

The relative influence of material rate-sensitivity and micro-inertia is further is illustrated in Fig. 4, which presents the evolution with the shock stress $P_{H}$ of the peak values of the viscous and dynamic stresses (maximum positive value for the dynamic stress) reached during the compression of the foam. It appears that micro-inertia effects become predominant when the shock magnitude increases.

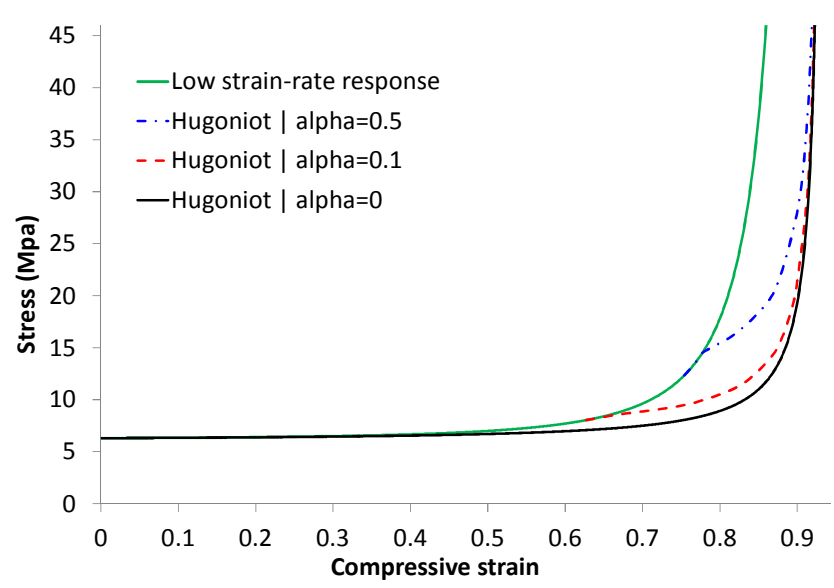

Fig. 2. Hugoniot stress-strain curve of a viscoplastic foam for several values of the parameter $\alpha$ characterising the material rate-sensitivity. The low-strain rate response of the foam (given by $P_{11}^{c R I}$, see Eq. (22)) is also plotted.

(a)

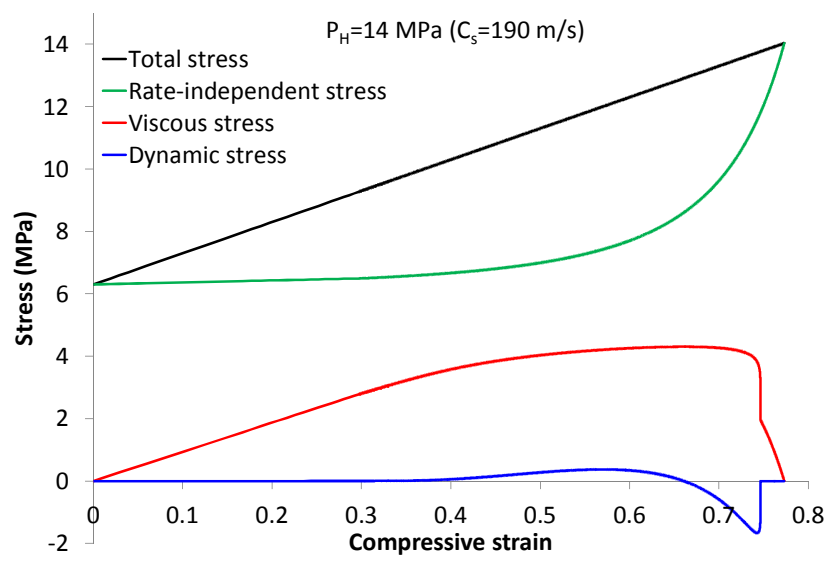

(b)

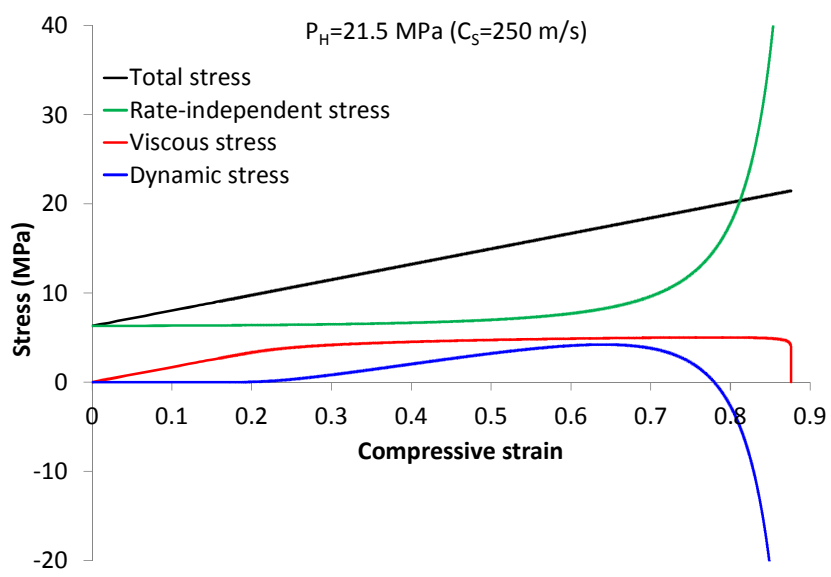

Fig. 3. Constitutive response of a viscoplastic foam $(\alpha=0.5)$ during a shock compression. The evolution of the total stress (Rayleigh line), the rate-independent stress, the viscous stress and the dynamic stress (see Eqs. (18) and (22)) with the nominal strain is presented for two shock magnitudes: (a) $P_{H}=14 \mathrm{MPa}$; (b) $P_{H}=21.5 \mathrm{MPa}$. 


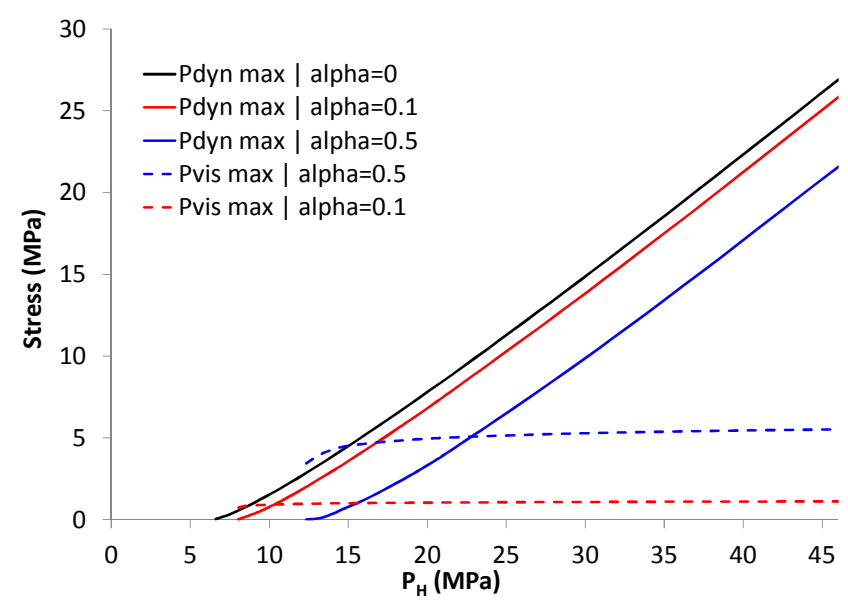

Fig. 4. Evolution with the shock magnitude of the peak values of the viscous stress and the dynamic stress reached during the shock compaction.

Micro-inertia effects are related to $R_{c}$, the characteristic radius of the cells of the foam microstructure (see Eq. (18)). We investigated the influence of $R_{c}$ on the shock response of a viscoplastic foam. Fig. 5 presents the Hugoniot curves computed for several values of the cell radius $\left(R_{c}=1.65 \mathrm{~mm}, R_{c}=2.5\right.$ $\mathrm{mm}$ and $R_{c}=3.3 \mathrm{~mm}$ ). It can be observed that $R_{c}$ has nearly no influence on the Hugoniot curve. This finding may seem paradoxical as it was shown previously that micro-inertia plays an important role in the foam behaviour at high shock magnitude. To understand this phenomenon, let us analyse the influence of $R_{c}$ on the shock structure. Fig. 6 displays of the strain $\left(\varepsilon_{11}^{c}\right)$, strainrate $\left(\dot{\varepsilon}_{11}^{c}\right)$ and strain-rate time derivative $\left(\ddot{\varepsilon}_{11}^{c}\right)$ profiles for several values of $R_{c}$ (for a shock magnitude of 21.5 $\mathrm{MPa}$ ). When $R_{c}$ is increased, the width of the shock layer becomes larger. The broadening of the shock front has for effect a reduction of the values of strain-rate and strain-rate time derivative achieved during the shock compression. Moreover, the decrease in the strain-rate time derivative is more pronounced. As a consequence, an increase of the cell radius does not lead to an increase of the peak value of the dynamic stress reached during the shock compression (Fig. 7) and the relative importance of viscous and micro-inertia effects remains almost unchanged.

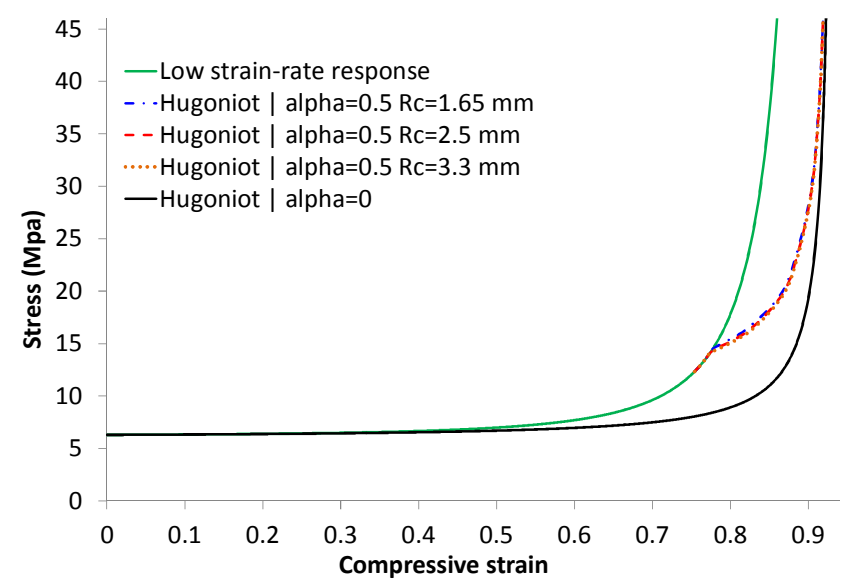

Fig. 5. Influence of the characteristic cell radius $R_{c}$ on the Hugoniot stress-strain curve of a viscoplastic foam (for $\alpha=0.5$ ). The low-strain rate response of the foam (given by $P_{11}^{c R I}$, see Eq. (22)) and the Hugoniot in absence of material rate-sensitivity $(\alpha=0)$ are also plotted.

(a)

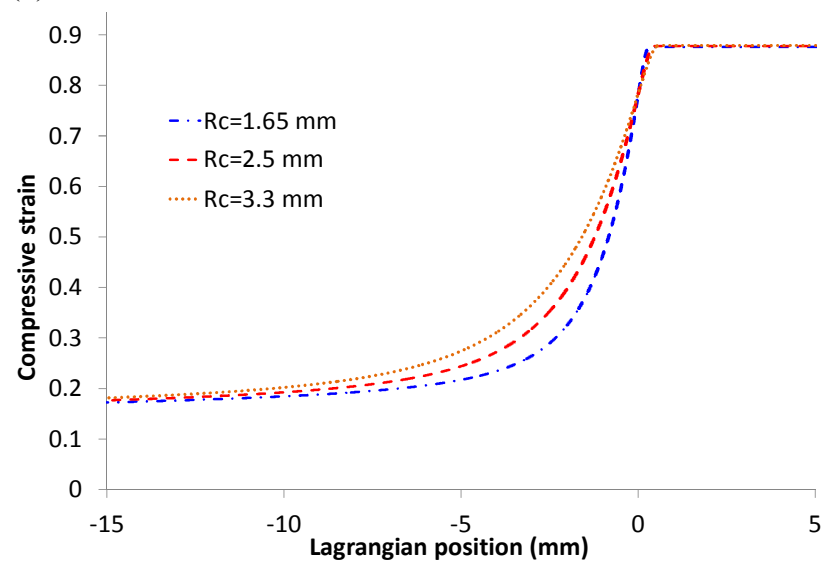

(b)

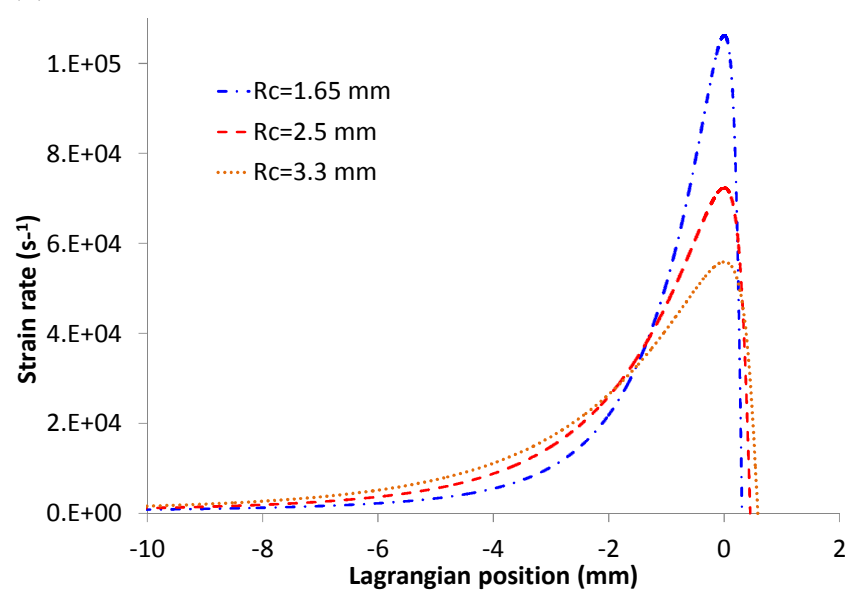

(c)

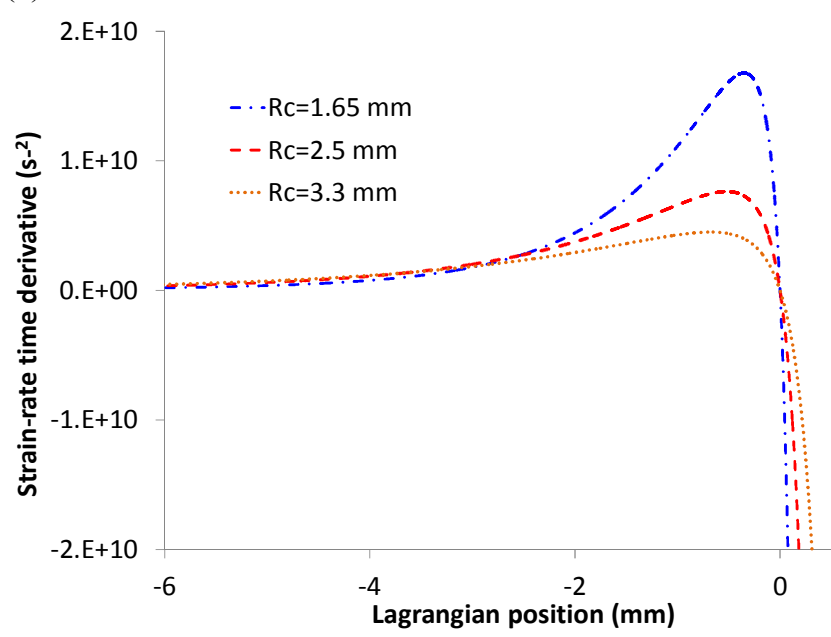

Fig. 6. Influence of the characteristic cell radius $R_{c}$ on the structure of a shock wave in a viscoplastic foam $(\alpha=0.5)$. The computations were carried out for $C_{S}=250 \mathrm{~m} / \mathrm{s}$ (corresponding to a shock magnitude $P_{H}$ of about $21.5 \mathrm{MPa}$ in all cases) (a) Nominal compressive strain versus position; (b) Strain-rate versus position; (c) Strain-rate time derivative versus position. 


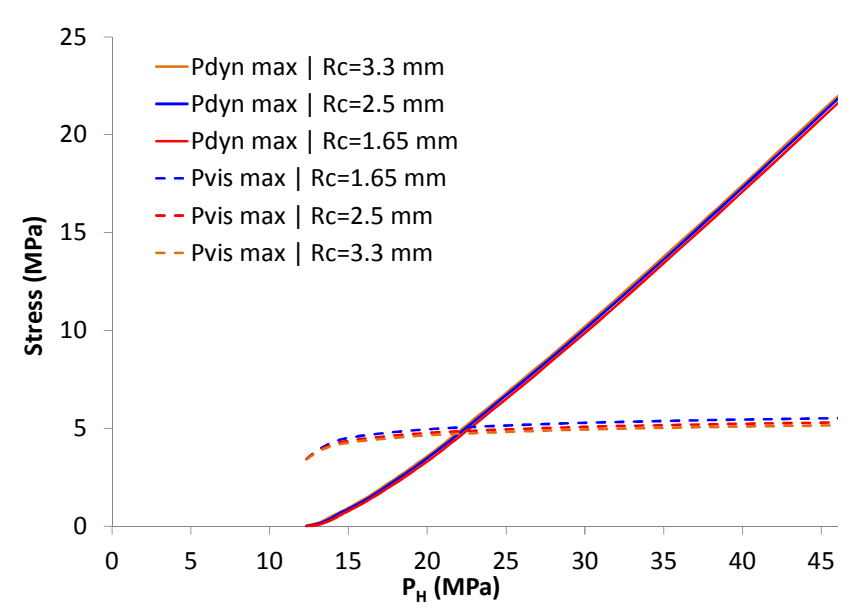

Fig. 7. Evolution with the shock magnitude of the peak values of the viscous stress and the dynamic stress reached during the shock compaction for several values of the characteristic cell radius $R_{c}$ (for $\alpha=0.5$ ).

\section{Conclusions}

The present work deals with the development of continuum models for the shock behaviour of foams and cellular solids. Of central importance in the proposed constitutive framework is the role played by microinertia effects. During the propagation of a shock wave, the rapid crushing of the cells of the foam microstructure generates local inertial effects, which affect the macroscopic foam response. Micro-inertia effects are taken into account using homogenisation techniques and lead to an additional contribution of the macrostress, named dynamic (or inertial) stress.

A closed-form expression of the Hugoniot stressstrain curve has been obtained for cellular solids made of a rate-insensitive base material. The predictions of this analytical model are in good agreement with experimental data for open-cell aluminium foams.

In the case of viscoplastic foams, the Hugoniot (and the shock structure) can be obtained via the numerical integration of a single differential equation. Our results show that, for weak shocks, the foam behaviour is controlled by viscous effects. Micro-inertia effects are enhanced when the shock magnitude increases and become predominant for strong shocks.

The work reported was supported by the "Association Nationale de la Recherche et de la Technologie" (ANRT) through the grant CIFRE no 0843/2013.

\section{References}

1. Y. Sun, Q.M. Li, Int. J. Impact Eng. 112, 74-115 (2017)

2. S. Pattofatto, I. Elnasri, H. Zhao, H. Tsitsiris, F. Hild, Y. Girard, J. Mech. Phys. Solids 55, 26722686 (2007)

3. A.T. Barnes, K. Ravi-Chandar, S. Kyriakides, S. Gaitanaros, Int. J. Solids Struct. 51, 1631-1645 (2014)
4. Z. Zheng, C. Wang, J. Yu, S.R. Reid, J.J. Harrigan, J. Mech. Phys. Solids 72, 93-114 (2014)

5. S. Gaitanaros, S. Kyriakides, Int. J. Solids Struct. 51, 1646-1661 (2014)

6. Y. Sun, Q.M. Li, S.A. McDonald, Mech. Mater. 99, 26-36 (2016)

7. P.J. Tan, J.J. Harrigan, S.R. Reid, Mater. Sci. Technol. 18, 480-488 (2002)

8. H. Zhao, I. Elnasri, S. Abdennadher, Int. J. Mech. Sci. 47, 757-774 (2005)

9. R. Barthélémy, N. Jacques, S. Kerampran, F. Vermeersch, Int. J. Solids Struct. 97, 445-457 (2016)

10. N. Jacques, R. Barthélémy, Int. J. Impact Eng. 115, 76-80 (2018)

11. V.S. Deshpande, N.A. Fleck, Int. J. Impact Eng. 24, 277-298 (2000)

12. A. Molinari, S. Mercier, J. Mech. Phys. Solids 49, 1497-1516 (2001)

13. S.Z. Dunin, V.V Surkov, J. Appl. Mech. Tech. Phys. 20, 612-618 (1979)

14. C. Czarnota, A. Molinari, S. Mercier, J. Mech. Phys. Solids 107, 204-228 (2017) 